(C) 2019 by the Arizona Board of Regents on behalf of the University of Arizona. This is an Open Access article, distributed under the terms of the Creative Commons Attribution-NonCommercial-ShareAlike licence (http://creativecommons.org/licenses/by-nc-sa/4.0/), which permits non-commercial re-use, distribution, and reproduction in any medium, provided the same Creative Commons licence is included and the original work is properly cited. The written permission of Cambridge University Press must be obtained for commercial re-use.

\title{
NEW RADIOCARBON DATES FOR THE LATE GRAVETTIAN IN EASTERN CENTRAL EUROPE
}

Jarosław Wilczyński ${ }^{1, *}$ • Tomasz Goslar ${ }^{2,3}$ • Piotr Wojtal ${ }^{1} \cdot$ Martin Oliva $^{4}$ • Ursula B Göhlich ${ }^{5} \cdot$ Walpurga Antl-Weiser ${ }^{5}$ • Petr Sída ${ }^{6,7}$ • Alexander Verpoorte ${ }^{8}$ • György Lengyel ${ }^{1}$

${ }^{1}$ Institute of Systematics and Evolution of Animals, Polish Academy of Sciences, Sławkowska 17, 31-016 Kraków, Poland

${ }^{2}$ Faculty of Physics, Adam Mickiewicz University, Umultowska 85, 61-614 Poznan, Poland

${ }^{3}$ Poznan Radiocarbon Laboratory, Foundation of the A. Mickiewicz University, Rubiez 46, 61-612 Poznan, Poland

${ }^{4}$ Anthropos Institute, Moravian Museum, Zelný trh 6, 65937 Brno, Czech Republic

${ }^{5}$ Natural History Museum Vienna, Burgring 7 A-1010 Vienna, Austria

${ }^{6}$ Institute of Archaeology of the CAS, 363/19 Čechyňská 60200, Brno, Czech Republic

${ }^{7}$ Department of Anthropology, Faculty of Science, Masaryk University, Kotlárská 2, 61137 Brno, Czech Republic

${ }^{8}$ Faculty of Archaeology, Leiden University, P.O. Box 9514, 2300 RA Leiden, The Netherlands

ABSTRACT. The Middle Upper Palaeolithic (MUP) in eastern Central Europe (ECE) comprises three variants of Gravettian culture: Early Gravettian, Pavlovian, and Late Gravettian. While Early Gravettian and Pavlovian are merely located in Lower Austria and Moravia, the Late Gravettian occupations occurred over the entire territory of ECE. Compared to the number of sites the radiocarbon dating and the absolute chronology of the Late Gravettian is rather poor. The results presented here bring a new set of radiocarbon $\left({ }^{14} \mathrm{C}\right)$ dates for the Late Gravettian period in ECE and propose that this period began and ended earlier than previously suggested.

KEYWORDS: chronology, human occupation, revision, Upper Paleolithic, Willendorf-Kostenkian.

\section{INTRODUCTION}

This report presents new accelerator mass spectrometry radiocarbon $\left(\mathrm{AMS}{ }^{14} \mathrm{C}\right)$ dates from six Late Gravettian sites (Figure 1). The Late Gravettian ( 29-24 ka cal BP), formerly often called Willendorf-Kostenkian, is a phase of the Gravettian culture in Eastern Central Europe (ECE), which is roughly dated to between 34 and $24 \mathrm{ka}$ cal BP. The Gravettian chronology begins with the Early Gravettian ( 34-30 ka cal BP) and its second phase is the Pavlovian ( $\sim 31-29 \mathrm{ka}$ cal BP) that directly precedes the Late Gravettian (Moreau 2009; Svoboda 2007, 2016). The Late Gravettian ends when the terrestrial ice sheet covering Europe in the last glacial Weichselian period reached its maximum extent at the Last Glacial Maximum (LGM) (Lengyel and Wilczyński 2018).

Early Gravettian sites are rare in ECE. The only clear example of this phase was found at Willendorf II layer 5, Wachau, Lower Austria (Moreau 2012). Another site, Dolní Věstonice IIa, Moravia (Czech Republic), produced material dated to the time of Early Gravettian (33-32 ka cal BP) (Svoboda 2016), but the lithic tools are typologically undiagnostic for Early Gravettian (Novák 2016). Other sites of this phase are found elsewhere in the Upper Danube basin and the Swabian Alps in western Central Europe (Moreau 2009).

Pavlovian sites have been recorded only in Lower Austria and Moravia (Czech Republic) (Oliva 2007; Svoboda 2007; Händel 2017), on the western margin of ECE. They occur

\footnotetext{
*Corresponding author. Email: wilczynski@isez.pan.krakow.pl.
} 


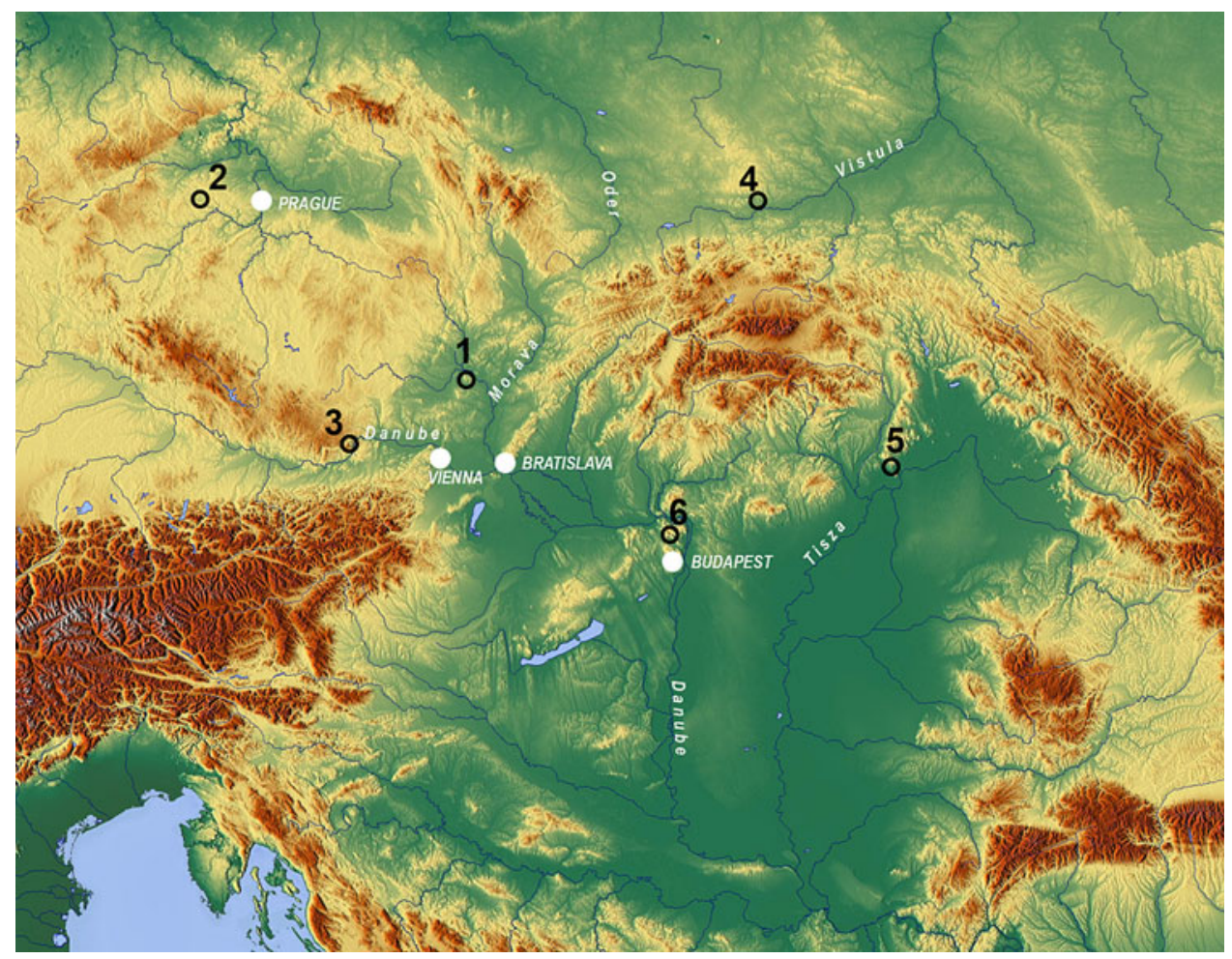

Figure 1 Location of the sites dated. 1 - Milovice I, 2 - Lubná VI, 3 - Willendorf II, 4 - Kraków Spadzista, 5 - Bodrogkeresztúr, 6 - Pilisszántó I.

much more frequently than sites of the preceding Early Gravettian in ECE. Most of the known locations are found in southern Moravia, especially on the northern slopes of the Pavlov Hills.

In contrast to the Early Gravettian and the Pavlovian, Late Gravettian occupations have been found all over ECE, which has yielded the most plentiful archaeological record of the Middle Upper Palaeolithic (MUP) (Kozłowski 2013; Lengyel 2016; Lengyel and Wilczyński 2018). Because of varying degrees of site preservation, the radiocarbon dates from all Late Gravettian sites tend to span a wider temporal period between $\sim 29$ and $24 \mathrm{ka}$ cal BP. In the existing MUP radiocarbon dataset, the end of the Pavlovian overlaps the beginning of the Late Gravettian. While almost all the Pavlovian sites are finely dated (Svoboda 2016), there are still inconsistencies in the chronology of the Late Gravettian (Lengyel 2008-2009; Oliva 2009) even at sites dated by a number of radiocarbon measurements (Wilczyński et al. 2012). Another problem is a lack of age determinations for some occupations. Therefore, efforts to refine the radiocarbon chronology for Late Gravettian occupations are particularly important, especially because of temporal overlap with the Pavlovian.

\section{METHODS}

All the dated samples reported here come from archeological occupations. We selected animal bones with taxonomic identification, unambiguous context, and modifications indicating actions by humans, such as stone tool cut marks and fresh bone breakage (green breaks). 
While animal bones in open-air sites associated with lithic artifacts are generally considered to be the results of human activity, accumulations of Mammuthus primigenius subfossil skeletal parts in Central and eastern Europe sometimes have been interpreted as collections made from naturally deceased animals (e.g., Steenstrup 1889; Wankel 1890; Absolon 1945; Kozłowski et al. 1974; Klima 1963; Haynes 1991; Soffer 1993; Svoboda et al. 2005; Wojtal and Sobczyk 2005; Oliva 2009). No consensus has been reached about the origins of sites yielding large amount of mammoth bones, but recent evidence strongly suggests that Gravettian hunters were able to kill mammoths with stone-tipped weapons (Kufel-Diakowska et al. 2016; Nuzhnyi et al. 2016; Sinitsyn et al. 2019; Wojtal et al. 2019). Mammoth bone accumulations that have been taphonomically analyzed allow reconstruction of butchering activity based on cut marks and green-bone breaks. One of the seven sites examined here is a rock shelter, Pilisszántó I Rockshelter, where the animal bone assemblage could have been accumulated during both human occupations and denning by carnivores.

AMS ${ }^{14} \mathrm{C}$ dating was performed at Poznań Radiocarbon Laboratory. All samples are from faunal remains. Collagen extraction was performed using the procedures described by Longin (1971) with further modifications (Piotrowska and Goslar 2002). Before extraction, the degree of collagen degradation was preliminarily checked by measuring content of $\mathrm{N}$ and $\mathrm{C}$ in bone using analyzer Flash EA 1112 Series. Usually the samples were forwarded for collagen extraction if $\mathrm{N}$ content in bone was not lower than $0.3 \%$. The bones were crushed mechanically to granulation $<0.3 \mathrm{~mm}$. The bone powder was treated with $2 \mathrm{M} \mathrm{HCl}$ at room temperature for 20 minutes, and $0.1 \mathrm{M} \mathrm{NaOH}$ at room temperature for 1 hour. After each step of treatment, the sample was centrifuged and the residuum was collected. Extraction of collagen was processed in $\mathrm{HCl}\left(\mathrm{pH}=3,80^{\circ} \mathrm{C}, 10 \mathrm{hr}\right)$, and after centrifugation the residuum was removed. The extracted collagen was ultrafiltered on pre-cleaned Vivaspin 15 MWCO 30 kD filters (Bronk Ramsey et al. 2004). Collagen quality was ultimately assessed by $\mathrm{C} / \mathrm{N}$ atomic ratio with interval of acceptance between 2.9 and 3.5 (van Klinken 1999). The obtained collagens were combusted in closed quartz tubes under vacuum together with $\mathrm{CuO}$ and $\mathrm{Ag}$ wool under $900^{\circ} \mathrm{C}$ over 10 hours. The obtained gas $\left(\mathrm{CO}_{2}+\right.$ water vapor) was then dried in a vacuum line and reduced with $\mathrm{H}_{2}$ using $2 \mathrm{mg}$ of $\mathrm{Fe}$ powder as a catalyst.

Bones charred to black included low nitrogen content and their $\mathrm{C} / \mathrm{N}$ ratio was over the limit of acceptance. These bones were pretreated using classical AAA method (Brock et al. 2010), and then combusted and processed further in the same way as the collagens.

The content of ${ }^{14} \mathrm{C}$ was measured using the NEC-produced "Compact Carbon AMS" (Goslar et al. 2004), by comparing intensities of ionic beams of ${ }^{14} \mathrm{C},{ }^{13} \mathrm{C}$, and ${ }^{12} \mathrm{C}$ measured for each sample and for standard samples (modern standard: "Oxalic Acid II" and ${ }^{14} \mathrm{C}$-free carbon: "background").

The conventional ${ }^{14} \mathrm{C}$ age was calculated using correction for isotopic fractionation (Stuiver and Polach 1977), basing on ${ }^{13} \mathrm{C} /{ }^{12} \mathrm{C}$ measured in the AMS spectrometer simultaneously with the ${ }^{14} \mathrm{C} /{ }^{12} \mathrm{C}$. Uncertainty of calculated ${ }^{14} \mathrm{C}$ age was determined using uncertainty implied from counting statistics, and also standard deviation of partial ${ }^{14} \mathrm{C} /{ }^{12} \mathrm{C}$ results, whichever was bigger. Uncertainties of ${ }^{14} \mathrm{C} /{ }^{12} \mathrm{C}$ ratios measured on standard and background samples were additionally taken into account. Calibration of ${ }^{14} \mathrm{C}$ age was performed against IntCal13 (Reimer et al. 2013) with OxCal ver. 4.3.2 (Bronk Ramsey and Lee 2013). We used the outlier analysis function of OxCal 4.3.2 (Bronk Ramsey 2009) to 
Table 1 Samples from Milovice I sector G.

\begin{tabular}{llllll}
\hline Serial no. & Origin & Archaeological feature & Year & Taxon & Specimen \\
\hline 1 & Sector G, square H & Archaeological layer & 1987 & Mammuthus $p$. & Molar \\
2 & Sector G, square L & Archaeological layer & 1981 & Mammuthus $p$ & Molar \\
3 & Sector G, square G & Archaeological layer & 1982 & Mammuthus $p$ & Molar \\
4 & Sector G, square H & Archaeological layer & 1986 & Mammuthus $p$. & Molar \\
\hline
\end{tabular}

determine the most probable age of the human occupation. This dating procedure was based on the Oxford Radiocarbon Accelerator Unit methodology.

\section{MATERIALS}

\section{Milovice I Sector G}

Located in Moravia, Czech Republic $\left(48^{\circ} 50^{\prime} 36^{\prime \prime} \mathrm{N} ; 16^{\circ} 41^{\prime} 21^{\prime \prime} \mathrm{E}\right)$, Milovice I is a Late Gravettian mammoth hunter camp near Dolní Věstonice (Oliva 2009). The best conserved part of the human occupation was located in excavation sector $G$, which yielded a typical set of Late Gravettian tool types. The archeological layer in sector $G$ was found in a sequence of loess. This was dated formerly on charcoals (species unidentified) to between 21.2 and $25.5 \mathrm{ka} \mathrm{BP}$, and one date was obtained on bone (species unidentified) to $24.1 \mathrm{ka}$ BP. All these dates were made with decay counting method.

Milovice I sector $\mathrm{G}$ yielded body parts of mammoth skeletons belonging to a minimum of 21 individuals (Oliva 2009). To ensure we dated different mammoth individuals, we selected only comparable mammoth molars from the collection stored at the Moravské zemské Museum, Brno (Table 1).

\section{Lubná VI}

Lubná yielded the first ever excavated paleolithic site in Bohemia (Czech Republic), dated to the Late Gravettian period (Verpoorte 2003; Šída 2016). There are eight sites identified to date at the outer periphery of the village Lubná. Lubná VI, located $48 \mathrm{~km}$ west of Prague $\left(50^{\circ} 04^{\prime} 52^{\prime \prime} \mathrm{N} ; 13^{\circ} 42^{\prime} 04^{\prime \prime} \mathrm{E}\right)$, was discovered in 2006 . It yielded hearths, lithics, and abundant faunal remains. The archaeological layer is embedded in loess, nearly $1.9 \mathrm{~m}$ below the recent ground surface. The archaeological bed is very distinct, up to a few $\mathrm{cm}$ in thickness, marked by a thin layer of stones, combustion features, faunal remains, and lithic tools. Reindeer bone fragments retrieved from the archaeological layer in the 2006 excavation at Lubná VI had earlier yielded three radiocarbon dates obtained by the Center for Isotope Research (University of Groningen, the Netherlands): 23,200 \pm 120 (GrA-57035), $23,070 \pm 120(\mathrm{GrA}-57037)$ and 23,150 \pm 110 (GrA-57076). The samples produced $\mathrm{C} / \mathrm{N}$ atomic ratios 4.16, 3.92 and 3.71, respectively, all of which exceeded the limit of acceptance 3.5 (van Klinken 1999). Values of $\delta^{15} \mathrm{~N}$ (1.94, 2.0 and 3.0, respectively) were low indicating degradation of bone collagen.

The new radiocarbon dates presented here were obtained from eight samples from the Lubná VI excavation in 2018 that continued recovering the layer found in 2006 (Table 2). The samples were reindeer remains and a single Capra ibex metatarsal bone fragment from the archaeological layer. 
Table 2 Samples from Lubná VI.

\begin{tabular}{llllll}
\hline $\begin{array}{l}\text { Serial } \\
\text { no. }\end{array}$ & Origin & Archaeological feature & Year & Taxon & Specimen \\
\hline 5 & Square D5 & Archaeological layer & 2018 & Rangifer tarandus & Metatarsus \\
6 & Square D5 & Archaeological layer & 2018 & Rangifer tarandus & Burned metapodium \\
7 & Square D5 & Archaeological layer & 2018 & Rangifer tarandus & Femur \\
8 & Square E7 & Archaeological layer & 2018 & Rangifer tarandus & Metatarsus \\
9 & Square F4 & Archaeological layer & 2018 & Rangifer tarandus & Metatarsus \\
10 & Square F5 & Archaeological layer & 2018 & Capra ibex & Metatarsus \\
11 & Square G4 & Archaeological layer & 2018 & Rangifer tarandus & Burned metatarsus \\
12 & Square G7 & Archaeological layer & 2018 & Rangifer tarandus & Mandibula \\
\hline
\end{tabular}

Table 3 Samples from Willendorf II layer 9.

\begin{tabular}{llllll}
\hline $\begin{array}{l}\text { Serial } \\
\text { no. }\end{array}$ & Origin & Archaeological feature & Year & Taxon & Specimen \\
\hline 13 & Layer 9 & Archaeological layer & $1908-09$ & Rangifer tarandus & Metapodium \\
14 & Layer 9 & Archaeological layer & $1908-09$ & Rangifer tarandus & Mandibula \\
15 & Layer 9 & Archaeological layer & $1908-09$ & Vulpes/Alopex sp. & Mandibula \\
16 & Layer 9 & Archaeological layer & $1908-09$ & Equus sp. & Molar \\
17 & Layer 9 & Archaeological layer & $1908-09$ & Vulpes/Alopex sp. & Mandibula \\
18 & Layer 9 & Archaeological layer & $1908-09$ & Equus sp. & Long bone \\
19 & Layer 9 & Archaeological layer & $1908-09$ & Mammuthus primigenius & Long bone \\
20 & Layer 9 & Archaeological layer & $1908-09$ & Rangifer tarandus & Astragalus \\
21 & Layer 9 & Archaeological layer & $1908-09$ & Mammuthus primigenius & Bone fragment \\
\hline
\end{tabular}

\section{Willendorf II Layer 9}

The site Willendorf II, located in Lower Austria $\left(48^{\circ} 19^{\prime} 23^{\prime \prime} \mathrm{N} ; 15^{\circ} 24^{\prime} 15^{\prime \prime} \mathrm{E}\right)$, yielded the longest Upper Paleolithic archeological sequence in Central Europe, dated between 42.5 and $27.2 \mathrm{ka}$ cal BP (Haesaerts et al. 1996; Nigst et al. 2014). Layer 9 is the uppermost in the series of the layers numbered from bottom to top. Previous radiocarbon dates on bones are between 29.3 and $27.2 \mathrm{ka}$ cal BP from the 1920s excavations. This layer is the westernmost archaeological record of the Late Gravettian that contains shouldered points, marking a sub-phase called Willendorf-Kostenkian (Kozłowski 2008), which yielded the famous Venus of Willendorf carved out of limestone (Antl-Weiser 2009).

Nine samples were selected from the bones of the 1908-1909 excavation stored at the Natural History Museum, Vienna (Table 3). The sampled species are mammoth, reindeer, fox, and horse. The bones were heavily impregnated with organic glue.

\section{Kraków Spadzista Layer 6}

The site has long been known as a mammoth hunting site (Kozłowski et al. 1974; Wojtal and Sobczyk 2005; Wilczyński et al. 2012), located in the city of Kraków, Lesser Poland $\left(50^{\circ} 03^{\prime} 22^{\prime \prime} \mathrm{N} ; 1^{\circ} 53^{\prime} 50^{\prime \prime} \mathrm{E}\right)$. The archaeological sequence includes Aurignacian, Late Gravettian, and Epigravettian occupations (Wilczyński 2007). Most abundant is the Late 
Table 4 Samples from Kraków Spadzista layer 6.

\begin{tabular}{|c|c|c|c|c|c|}
\hline Serial no. & Origin & $\begin{array}{l}\text { Archaeological } \\
\text { feature }\end{array}$ & Year & Taxon & Specimen \\
\hline 22 & Area $\mathrm{B}+\mathrm{B} 1$, layer 6 & $\begin{array}{l}\text { Archaeological } \\
\text { layer }\end{array}$ & $1995-2002$ & $\begin{array}{l}\text { Mammuthus } \\
\text { primigenius }\end{array}$ & $\begin{array}{l}\text { Bone } \\
\text { fragment }\end{array}$ \\
\hline 23 & Area B+B1, layer 6 & $\begin{array}{l}\text { Archaeological } \\
\text { layer }\end{array}$ & 1995-2002 & $\begin{array}{l}\text { Mammuthus } \\
\text { primigenius }\end{array}$ & $\begin{array}{l}\text { Bone } \\
\text { fragment }\end{array}$ \\
\hline 24 & Area B+B1, layer 6 & $\begin{array}{l}\text { Archaeological } \\
\text { layer }\end{array}$ & 1995-2002 & $\begin{array}{l}\text { Mammuthus } \\
\text { primigenius }\end{array}$ & $\begin{array}{l}\text { Bone } \\
\text { fragment }\end{array}$ \\
\hline 25 & Area B+B1, layer 6 & $\begin{array}{l}\text { Archaeological } \\
\text { layer }\end{array}$ & 1995-2002 & $\begin{array}{l}\text { Mammuthus } \\
\text { primigenius }\end{array}$ & Rib \\
\hline 26 & Area B+B1, layer 6 & $\begin{array}{l}\text { Archaeological } \\
\text { layer }\end{array}$ & 1995-2002 & $\begin{array}{l}\text { Mammuthus } \\
\text { primigenius }\end{array}$ & $\begin{array}{l}\text { Burned } \\
\text { bone }\end{array}$ \\
\hline 27 & Area B+B1, layer 6 & $\begin{array}{l}\text { Archaeological } \\
\text { layer }\end{array}$ & 1995-2002 & $\begin{array}{l}\text { Mammuthus } \\
\text { primigenius }\end{array}$ & $\begin{array}{l}\text { Bone } \\
\text { fragment }\end{array}$ \\
\hline 28 & Area $B+B 1$, layer 6 & $\begin{array}{l}\text { Archaeological } \\
\text { layer }\end{array}$ & 1995-2002 & $\begin{array}{l}\text { Mammuthus } \\
\text { primigenius }\end{array}$ & $\begin{array}{l}\text { Bone } \\
\text { fragment }\end{array}$ \\
\hline 29 & Area B+B1, layer 6 & $\begin{array}{l}\text { Archaeological } \\
\text { layer }\end{array}$ & 1995-2002 & $\begin{array}{l}\text { Mammuthus } \\
\text { primigenius }\end{array}$ & $\begin{array}{l}\text { Bone } \\
\text { fragment }\end{array}$ \\
\hline 30 & Area B+B1, layer 6 & $\begin{array}{l}\text { Archaeological } \\
\text { layer }\end{array}$ & 1995-2002 & $\begin{array}{l}\text { Mammuthus } \\
\text { primigenius }\end{array}$ & $\begin{array}{l}\text { Bone } \\
\text { fragment }\end{array}$ \\
\hline 31 & Area B+B1, layer 6 & $\begin{array}{l}\text { Archaeological } \\
\text { layer }\end{array}$ & 1995-2002 & $\begin{array}{l}\text { Mammuthus } \\
\text { primigenius }\end{array}$ & $\begin{array}{l}\text { Thoracic } \\
\text { vertebra }\end{array}$ \\
\hline 32 & Area B+B1, layer 6 & $\begin{array}{l}\text { Archaeological } \\
\text { layer }\end{array}$ & 1995-2002 & $\begin{array}{l}\text { Mammuthus } \\
\text { primigenius }\end{array}$ & $\begin{array}{l}\text { Bone } \\
\text { fragment }\end{array}$ \\
\hline 33 & $\begin{array}{l}\text { Area B2, uppermost } \\
\text { level of layer } 6\end{array}$ & $\begin{array}{l}\text { Archaeological } \\
\text { layer }\end{array}$ & 2016 & $\begin{array}{l}\text { Mammuthus } \\
\text { primigenius }\end{array}$ & $\begin{array}{l}\text { Bone } \\
\text { fragment }\end{array}$ \\
\hline 34 & Trench B2, layer 6 & $\begin{array}{l}\text { Archaeological } \\
\text { layer }\end{array}$ & 2016 & $\begin{array}{l}\text { Mammuthus } \\
\text { primigenius }\end{array}$ & $\begin{array}{l}\text { Bone } \\
\text { fragment }\end{array}$ \\
\hline 35 & Trench B3, layer 6 & $\begin{array}{l}\text { Archaeological } \\
\text { layer }\end{array}$ & 2016 & $\begin{array}{l}\text { Mammuthus } \\
\text { primigenius }\end{array}$ & $\begin{array}{l}\text { Bone } \\
\text { fragment }\end{array}$ \\
\hline 36 & Trench B3, layer 6 & $\begin{array}{l}\text { Archaeological } \\
\text { layer }\end{array}$ & 2016 & $\begin{array}{l}\text { Mammuthus } \\
\text { primigenius }\end{array}$ & $\begin{array}{l}\text { Bone } \\
\text { fragment }\end{array}$ \\
\hline 37 & Trench B3, layer 6 & $\begin{array}{l}\text { Archaeological } \\
\text { layer }\end{array}$ & 2016 & $\begin{array}{l}\text { Mammuthus } \\
\text { primigenius }\end{array}$ & Rib \\
\hline 38 & Trench B3, layer 6 & $\begin{array}{l}\text { Archaeological } \\
\text { layer }\end{array}$ & 2016 & $\begin{array}{l}\text { Mammuthus } \\
\text { primigenius }\end{array}$ & Tooth \\
\hline
\end{tabular}

Gravettian in layer 6. The layer was severely disturbed by periglacial events. Former radiocarbon dates $(n=26)$ for the Late Gravettian occupation range between 30.3 and 24.5 ka cal BP (Wojtal et al. 2015; Wilczyński et al. 2015).

The 18 samples dated here are all of mammoth material derived from $\mathrm{B}+\mathrm{B} 1, \mathrm{~B} 2$ and $\mathrm{B} 3$ trenches located in the northern part of the site, where a large accumulation of bones was discovered (Kozłowski et al. 1974; Wojtal and Sobczyk 2005) (Table 4). The bones are stored at the Institute of Systematics and Evolution of Animals, Polish Academy of Sciences, Kraków. 
Table 5 Samples from Bodrogkeresztúr.

\begin{tabular}{llllll}
\hline Serial no. & Origin & Archaeological feature & Year & Taxon & Specimen \\
\hline 39 & A & Archaeological layer & 1963 & $\begin{array}{c}\text { Equus sp. } \\
\text { Alces alces }\end{array}$ & Mpper tooth \\
41 & A 36-39 & Archaeological layer & 1963 & $\begin{array}{l}\text { Mandibula } \\
\text { Aammuthus } \\
\text { Frimigenius }\end{array}$ & Femur \\
42 & G 15 & Archaeological layer & 1963 & Alces alces & Mandibula \\
\hline
\end{tabular}

\section{Bodrogkeresztúr}

The site is located in northeast Hungary $\left(48^{\circ} 09^{\prime} 05^{\prime \prime} \mathrm{N} ; 21^{\circ} 20^{\prime} 49^{\prime \prime} \mathrm{E}\right)$, on a small hill called Henye, situated east of the village Bodrogkeresztúr. Two excavations were carried out at the site, one in 1963 (Vértes 1966) and another in 1982 (Dobosi 2000). The archaeological layer was embedded in loess (Dobosi 2000). A paleosol was noticed at the site, but the relation to the archaeological layer is controversial (Vértes 1966 vs. Dobosi 2000). There are four unreliable radiocarbon dates from the site (Lengyel 2008-2009). The one which is most often cited is $28,700 \pm 3,000$ (GXO-195) with a very large standard deviation. Another date, $26.3 \mathrm{ka} \mathrm{BP}$ (Deb-2555), was obtained from a bulk charcoal sample retrieved from a $5 \mathrm{~kg}$ sample of the paleosol. Two dates on bone samples, $18.5 \mathrm{ka} \mathrm{BP}$ (Deb-3381) and $10.6 \mathrm{ka}$ BP (Hv-12986), possibly were obtained from contaminated samples (Dobosi 2000; Dobosi and Szántó 2003). The site has been re-attributed to the Late Gravettian period on the basis of lithic tool typology (Lengyel 2015).

The four samples dated here derive from three species of the faunal collection of the 1963 excavation: mammoth, European elk, and horse (Table 5). The bones were stored at the Mining and Geological Survey of Hungary (formerly Institute of Geophysics and Geology of Hungary).

\section{Pilisszántó I Rockshelter}

The site is located in the Pilis Mountains, northern Hungary $\left(47^{\circ} 40^{\prime} 43^{\prime \prime} \mathrm{N} ; 18^{\circ} 52^{\prime} 52^{\prime \prime} \mathrm{E}\right)$. The site was extensively excavated in 1914-1915 (Kormos and Lambrecht 1915), then the rest of the sediment was removed in 1951 (Gábori 1954). The archaeological material is sparse but consists almost entirely of lithic hunting tools (Dobosi and Vörös 1987). According to the lithic tool typology the lower layer complex can be attributed to the Late Gravettian (Lengyel 2016). The middle and upper layer complexes contained culturally undiagnostic lithic tool types.

The site yielded abundant remains of reindeer (Kormos and Lambrecht 1915). The stratigraphy included seven "diluvial" (i.e. Pleistocene) layers grouped into three units: upper layer complex (D1-2), middle layer complex (D3-5) and lower layer complex (D6-7). The exact ages of the human occupations have been unknown and the chronology of the layers was estimated on the basis of the biostratigraphy. The upper and the middle layer complexes were dated to the "Pilisszántó faunal phase" approximately between 22 and $16 \mathrm{ka} \mathrm{BP,} \mathrm{the} \mathrm{Würm} \mathrm{III} \mathrm{cold}$ maximum (LLGM) which preceded the "Bajót faunal phase" 16-13 ka BP (Dobosi and Vörös 1987). The lower layer complex does not correspond to any of the known faunal phases (unnamed faunal phase in Dobosi and Vörös 1987). Later it was merged into the preceding Istállóskó faunal phase equal to the Upper Würm that immediately followed MIS3 
Table 6 Samples from Pilisszántó I rockshelter.

\begin{tabular}{lllcll}
\hline $\begin{array}{l}\text { Serial } \\
\text { no. }\end{array}$ & Origin & $\begin{array}{l}\text { Archaeological } \\
\text { feature }\end{array}$ & Year & Taxon & Specimen \\
\hline 43 & Lower layer complex & Geological unit & $1914-15$ & Rangifer tarandus & Phalanx \\
44 & Lower layer complex & Geological unit & $1914-15$ & Rangifer tarandus & Mandibula \\
\hline
\end{tabular}

Table 7 Milovice I sector $\mathrm{G}$ radiocarbon dates.

\begin{tabular}{lccccccccccc}
\hline \multicolumn{1}{c}{ Serial no. } & Lab no. & ${ }^{14} \mathrm{C}$ BP & \pm & \multicolumn{9}{c}{ cal BP } & $95.4 \%$ & $\% \mathrm{~N}$ & $\% \mathrm{C}$ & $\%$ coll & $\%$ Ncoll & $\%$ Ccoll & C/N \\
\hline 1 & Poz-99607 & 24900 & 200 & 29441 & 28537 & 1.1 & 5.4 & 1.8 & 16.9 & 46.5 & 3.21 \\
2 & Poz-99606 & 25770 & 210 & 30599 & 29429 & 1.9 & 6 & 6.4 & 18.9 & 51.6 & 3.19 \\
3 & Poz-99610 & 25880 & 220 & 30696 & 29525 & 1.4 & 6.4 & 3.8 & 18.2 & 49.8 & 3.19 \\
4 & Poz-99608 & 26010 & 230 & 30801 & 29635 & 3.4 & 9.7 & 5.2 & 17.9 & 48.6 & 3.17 \\
\hline
\end{tabular}

(Vörös 2000). This revision unseparated the Pilisszántó faunal phase from the Bajót faunal phase, and the middle and upper layer complexes were dated to the Pilisszántó-Bajót faunal phase (Vörös 2000). This division however was not used consistently. The age of the lower layer complex was also estimated to be as old as $23 \mathrm{ka} \mathrm{BP}$, the middle layer $21 \mathrm{ka} \mathrm{BP}$; the upper layer remained unclassified (Dobosi and Szántó 2003).

The two samples dated here are a reindeer mandible (Kormos and Lambrecht 1915: Figure 44) and a reindeer phalanx from the lower layer complex (Table 6).

\section{RESULTS}

The dating results are listed in Tables 7-12. Dates obtained on collagen of poor or not checked (n.a.) quality (displayed in italics) have been left uncalibrated and ignored from discussion. Charred bones from which collagen was not extracted are denoted with "AAA" in the " $\%$ coll" column. The serial numbers identify the samples listed in Tables 1-6.

\section{Milovice I Sector G}

Milovice I sector $\mathrm{G}$ dates (Table 7) correspond with what was obtained earlier from the site (Oliva 2009). The new dates between 30.8 and $28.5 \mathrm{ka}$ cal BP are contemporaneous with the Pavlovian, although the lithic assemblage of Milovice I sector G is clearly associated with the Late Gravettian. The formerly obtained 22-21 ka BP ages on unknown species of wood charcoals (ISGS-1691 and ISGS-1690) (Oliva 2009) seem too young for the Milovice I Late Gravettian occupation.

A taphonomic analysis of clustered mammoth bones concluded that the bone accumulation was fairly rapid, encompassing no more than few hundred years (Brugère and Fontana 2009). To reconcile this brief time span with our dating results, we tried to combine (Figure 2) probability distributions of calibrated dates, blurred by adding uniform distributions in 500 year intervals $(\mathrm{U}(-250,250))$. The process of combining generally assumes calendar dates of all 
Table 8 Lubná VI radiocarbon dates.

\begin{tabular}{lccccccccccc}
\hline Serial no. & Lab no. & BP & \pm & cal BP & $95.4 \%$ & $\% \mathrm{~N}$ & $\% \mathrm{C}$ & $\%$ coll & $\%$ Ncoll & $\%$ Ccoll & C/N \\
\hline 5 & Poz-107226 & 19720 & 110 & 24051 & 23446 & 1.3 & 6.9 & 3.3 & 18.2 & 50.0 & 3.21 \\
6 & Poz-107493 & 22820 & 160 & 27486 & 26696 & 0.5 & 7.0 & AAA & - & - & - \\
7 & Poz-107228 & 23010 & 140 & 27595 & 27060 & 1.2 & 9.5 & 3.8 & 16.9 & 46.6 & 3.22 \\
8 & Poz-107229 & 23260 & 130 & 27735 & 27291 & 1.0 & 8.2 & 4.2 & 17.4 & 47.5 & 3.18 \\
9 & Poz-107225 & 22860 & 130 & 27503 & 26861 & 1.3 & 6.5 & 2 & 16.5 & 45.0 & 3.18 \\
10 & Poz-107230 & 21190 & 120 & 25795 & 25239 & 1.5 & 8.3 & 3.4 & 17.1 & 46.6 & 3.17 \\
11 & Poz-107494 & 20060 & 120 & 24438 & 23822 & 0.5 & 7.0 & AAA & - & - & - \\
12 & Poz-107224 & 22550 & 130 & 27241 & 26487 & 0.6 & 6.6 & 1.5 & 15.9 & 43.5 & 3.19 \\
\hline
\end{tabular}

Table 9 Willendorf II layer 9 radiocarbon dates.

\begin{tabular}{|c|c|c|c|c|c|c|c|c|c|c|c|}
\hline Serial no. & Lab no. & BP & \pm & $\begin{array}{c}\text { cal } \\
95 .\end{array}$ & & $\% \mathrm{~N}$ & $\% \mathrm{C}$ & $\%$ coll & $\%$ Ncoll & $\%$ Ccoll & $\mathrm{C} / \mathrm{N}$ \\
\hline 13 & Poz-100126 & 10080 & 70 & - & - & 0.4 & 15.6 & 0.08 & n.a. & n.a. & n.a. \\
\hline 14 & Poz-99666 & 12910 & 100 & - & - & 2.0 & 7.7 & 5.6 & 16.1 & 52.7 & 3.82 \\
\hline 15 & Poz-99661 & 13270 & 100 & - & - & 0.5 & 4.8 & 1.0 & 15.2 & 48.5 & 3.72 \\
\hline 16 & Poz-99668 & 13350 & 100 & - & - & 1.2 & 5.4 & 2.2 & 16.5 & 52.9 & 3.74 \\
\hline 17 & Poz-99662 & 15690 & 130 & - & - & 0.8 & 5.7 & 2.8 & 16.9 & 50.9 & 3.51 \\
\hline 18 & Poz-99664 & 17130 & 140 & - & - & 2.4 & 7.2 & 7.1 & 16.9 & 49.9 & 3.44 \\
\hline 19 & Poz-99665 & 18370 & 180 & - & - & 0.6 & 3.4 & 0.9 & 16 & 46.1 & 3.36 \\
\hline 20 & Poz-99952 & 18400 & 100 & - & - & 0.5 & 5.2 & $A A A$ & - & - & - \\
\hline 21 & Poz-99994 & 21400 & 140 & - & - & 0.6 & 5.1 & $A A A$ & - & - & - \\
\hline
\end{tabular}

Table 10 Kraków Spadzista layer 6 radiocarbon dates.

\begin{tabular}{|c|c|c|c|c|c|c|c|c|c|c|c|}
\hline Serial no. & Lab no. & BP & \pm & $\begin{array}{c}\text { cal } \\
95 .\end{array}$ & & $\% \mathrm{~N}$ & $\% \mathrm{C}$ & $\%$ coll & $\%$ Ncoll & $\%$ Ccoll & $\mathrm{C} / \mathrm{N}$ \\
\hline 22 & Poz-58706 & 11390 & 130 & - & - & 0.4 & 1.9 & 0.1 & n.a. & n.a. & n.a. \\
\hline 23 & Poz-86236 & 17670 & 100 & - & - & 1.32 & 10.92 & 1.6 & 13.8 & 51.6 & 4.35 \\
\hline 24 & Poz-77076 & 17870 & 90 & - & - & 0.4 & 3.1 & 0.4 & n.a. & n.a. & n.a. \\
\hline 25 & Poz-100023 & 19720 & 200 & 24240 & 23220 & 0.5 & 5.6 & 1.7 & 18.1 & 49.4 & 3.18 \\
\hline 26 & Poz-67404 & 21700 & 120 & 26169 & 25736 & 0.2 & 7.6 & AAA & - & - & - \\
\hline 27 & Poz-86233 & 23370 & 130 & 27786 & 27368 & 0.47 & 3.69 & 1.1 & 16.7 & 47.8 & 3.33 \\
\hline 28 & Poz-86231 & 21710 & 110 & - & - & 0.83 & 6.86 & 1.5 & 14.4 & 49.7 & 4.02 \\
\hline 29 & Poz-86232 & 23570 & 140 & 27924 & 27467 & 0.99 & 4.95 & 2.0 & 17.8 & 48.5 & 3.19 \\
\hline 30 & Poz-86237 & 23850 & 200 & 28389 & 27604 & 1.2 & 6.78 & 2.7 & 17.4 & 46.7 & 3.14 \\
\hline 31 & Poz-77075 & 23970 & 160 & 28415 & 27715 & 1.8 & 6.5 & 0.7 & 14.1 & 38.8 & 3.22 \\
\hline 32 & Poz-67406 & 25830 & 190 & 30618 & 29515 & 0.7 & 5.2 & AAA & - & - & - \\
\hline 33 & Poz- 87042 & 18740 & 100 & 22879 & 22397 & 0.9 & 6.1 & 0.6 & 13.9 & 39.1 & 3.29 \\
\hline 34 & Poz-87043 & 22460 & 140 & 27186 & 26359 & 1.5 & 7.3 & 1.7 & 16.3 & 45.4 & 3.25 \\
\hline 35 & Poz- 86230 & 23440 & 140 & 27832 & 27398 & 1.11 & 6.21 & 0.4 & 13.3 & 37.1 & 3.25 \\
\hline 36 & Poz-86229 & 23710 & 150 & 28098 & 27543 & 0.4 & 4.39 & 3.0 & 15.4 & 42.2 & 3.20 \\
\hline 37 & Poz-101183 & 24200 & 250 & 28729 & 27778 & 0.6 & 6.3 & 0.3 & 16.2 & 44.2 & 3.18 \\
\hline 38 & Poz-101206 & 25120 & 320 & 30098 & 28525 & 3.4 & 10.4 & 10.6 & 18.6 & 49.8 & 3.12 \\
\hline
\end{tabular}


Table 11 Bodrogkeresztúr radiocarbon dates.

\begin{tabular}{lccccccccccc}
\hline Serial no. & Lab no. & BP & \pm & cal BP & $95.4 \%$ & $\% \mathrm{~N}$ & $\% \mathrm{C}$ & $\%$ coll & $\%$ Ncoll & $\%$ Ccoll & C/N \\
\hline 39 & Poz-95148 & 21270 & 190 & 25939 & 25203 & 0.6 & 4.2 & 0.4 & 16.1 & 42.6 & 3.09 \\
40 & Poz-95150 & 23010 & 220 & 27706 & 26833 & 0.4 & 8.6 & 2.1 & 17.1 & 47.4 & 3.23 \\
41 & Poz-95146 & 22620 & 210 & 27401 & 26416 & 0.7 & 4.7 & 3.5 & 17.3 & 47.8 & 3.22 \\
42 & Poz-95149 & 23380 & 230 & 27925 & 27229 & 1.7 & 7.0 & 4.5 & 18.5 & 51.8 & 3.27 \\
\hline
\end{tabular}

Table 12 Pilisszántó I rockshelter lower layer complex dates.

\begin{tabular}{llcccccccccc}
\hline Serial no. & Lab no. & BP & \pm & cal BP & $95.4 \%$ & $\% \mathrm{~N}$ & $\% \mathrm{C}$ & $\%$ coll & $\%$ Ncoll & $\%$ Ccoll & C/N \\
\hline 43 & Poz-95152 & 24990 & 270 & 29680 & 28480 & 2.0 & 7.0 & 3.4 & 16.2 & 45.4 & 3.27 \\
44 & Poz-95153 & 25150 & 280 & 29972 & 28596 & 2.3 & 9.5 & 5.8 & 17.9 & 49.1 & 3.20 \\
\hline
\end{tabular}

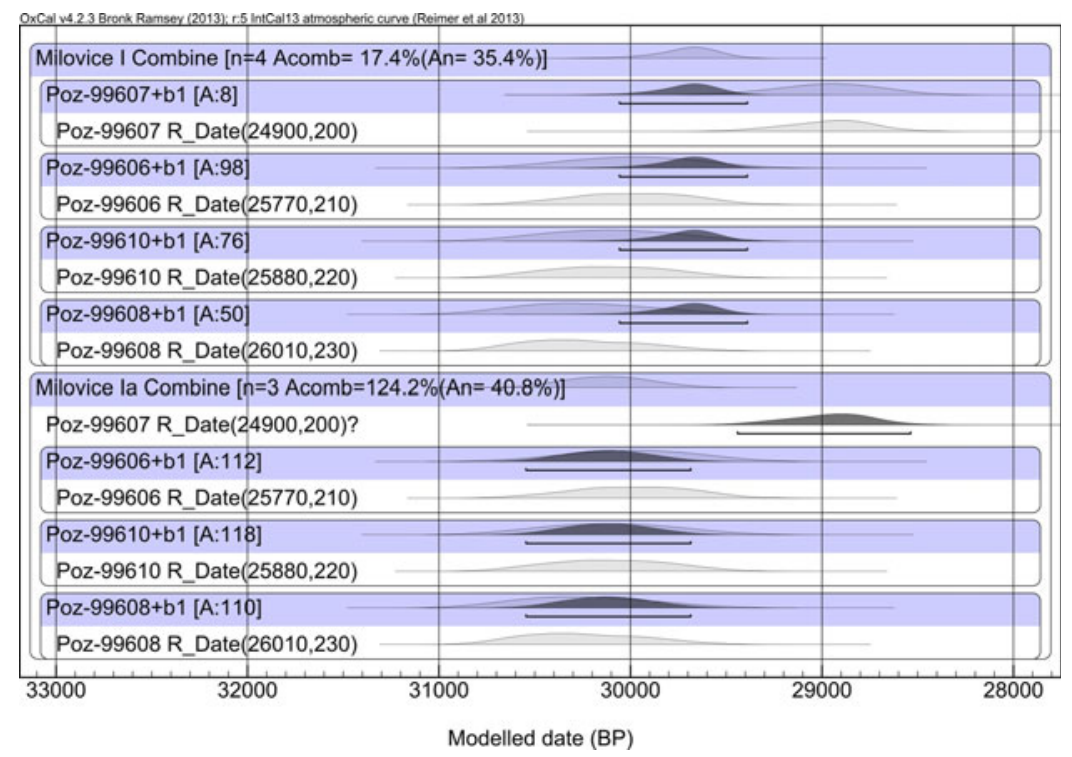

Figure 2 Upper part: combined calibrated ${ }^{14} \mathrm{C}$ dates from Milovice I sector $\mathrm{G}$, blurred by adding uniform probability distributions 500 years wide $(\mathrm{b} 1=\mathrm{U}(-250,250))$. Lower part: same as above, but with Poz-99607 treated as an outlier of the phase.

samples to be the same. Using blurred distributions allows the differing of combined actual dates from one another (here, the maximum allowed difference is set at $500 \mathrm{yr}$ ).

The trial to combine all 4 dates (Figure 2, upper part) was unsuccessful, as indicated by low value of Agreement index $(A c o m b=17.4 \%)$. A fully acceptable agreement index $($ Acomb $=124.2 \%)$ was obtained in the second trial, and the most offsetting date (Poz-99607) was declared as outlier (Figure 2, lower part). We therefore posit that the bones in the human occupation were accumulated probably 30.4 and $29.8 \mathrm{ka}$ cal BP (Figure 3). 


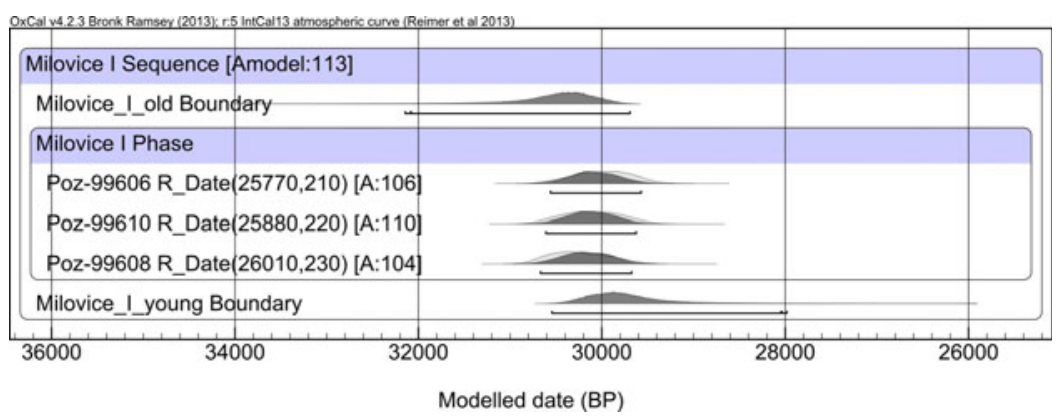

Figure 3 Bayesian modeling of phase encompassing dates of 3 bones from Milovice I sector $\mathrm{G}$.

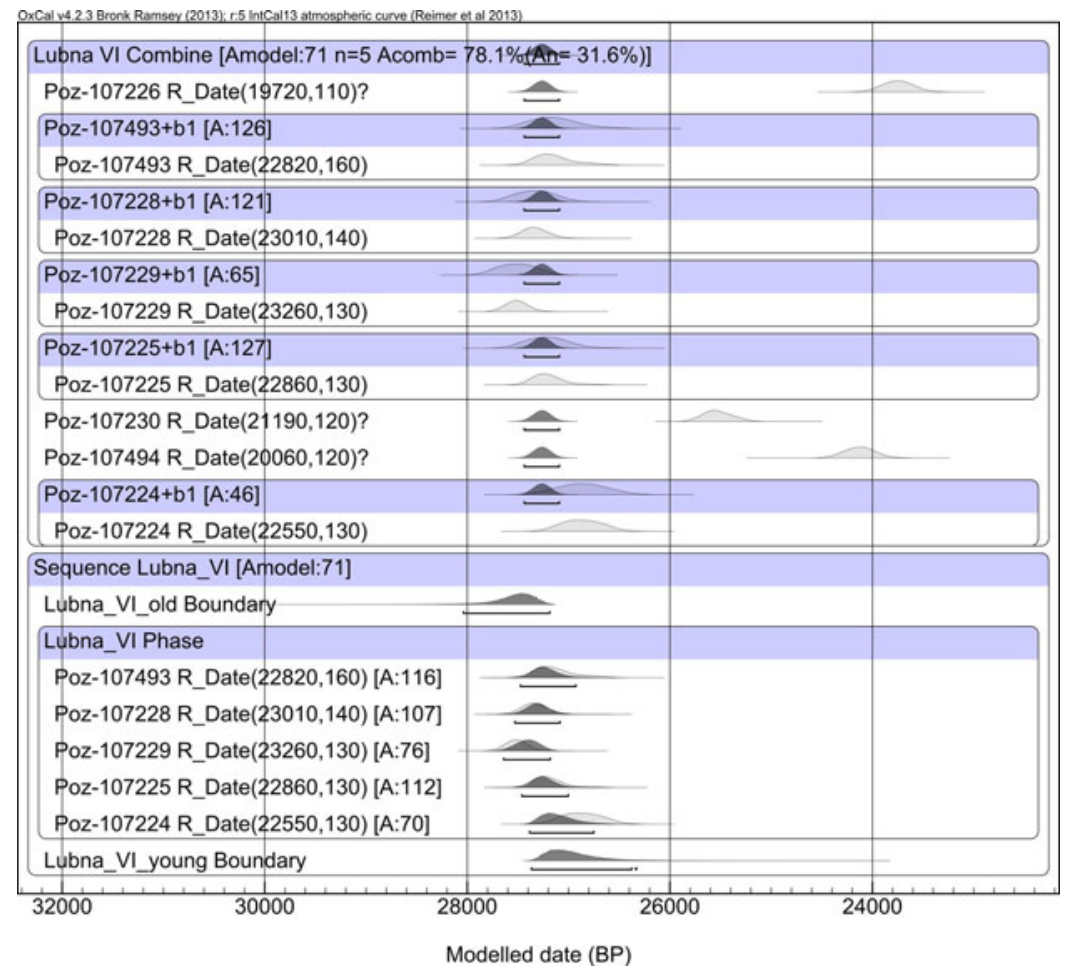

Figure 4 Upper part: combined calibrated ${ }^{14} \mathrm{C}$ dates from Lubná VI, blurred by adding uniform probability distributions 500 years wide $(\mathrm{b} 1=\mathrm{U}(-250,250))$. Three of eight dates which are clearly younger were declared outliers of the phase. Lower part: Bayesian modeling of phase encompassing dates of 5 bones.

\section{Lubná VI}

A small excavated area of the site (a dozen square meters) encompasses two hearths with numerous animal remains and stone artifacts, suggesting a brief period of human occupation. Indeed, four of eight analyzed bones (Table 8 ) have ${ }^{14} \mathrm{C}$ ages close to one 
another, and five of eight samples (Figure 4, upper part) appeared to derive from a phase not longer than 500 years (as demonstrated in a way similar to that applied to Milovice I), most probably dated between 27.5 and $27.1 \mathrm{ka}$ cal BP (Figure 4, lower part). Three other bones retrieved (Poz-107226, Poz-107230, and Poz-107494) are distinctly younger; however, only one date (Poz-107226) falls out of the expected Late Gravettian time interval. We point out that the cluster of our dates perfectly corresponds with the Groningen AMS dates obtained in 2006 from the same archaeological layer, although the quality of collagens which were ${ }^{14} \mathrm{C}$-analyzed in 2006 was distinctly poorer, as indicated by high $\mathrm{C} / \mathrm{N}$ ratios. Diverging radiocarbon dating results also might reflect repeated occupations at the same location over time (Kuzmin and Keates 2005); however, finds at Lubná VI solely were found in a very distinct single archaeological layer, below and above which no traces of human occupations occurred in the loess. Therefore, the reason for radiometric ages which are 2-3 millennia later than expected must stem from another yet-unrevealed source.

\section{Willendorf II Layer 9}

Eight of the nine Willendorf II layer 9 dates are not within the expected time range of the Late Gravettian archaeological period (Table 9); only one date obtained from a burnt mammoth bone fragment (Poz-99994) is within the range. The $\mathrm{C} / \mathrm{N}$ atomic ratios of four samples are over the 3.5 limit of acceptance, and although two other samples (Poz-99662 and Poz-99664) yielded $\mathrm{C} / \mathrm{N}$ ratios within the limit of acceptance, 3.44 and 3.36, respectively, these are clearly higher than $\mathrm{C} / \mathrm{N}$ ratios of most other collagens dated in this work. In one sample (Poz-100126), the low amount of extracted collagen did not allow for $\mathrm{C} / \mathrm{N}$ analysis, and two remaining dates were obtained on charred bones. It is probable that the glue impregnation of the bones in the early 20th century caused the erratic dating results. The glue was likely applied on most of the faunal assemblage, since the young dates were obtained from different species (horse, reindeer, fox, and the mammoth). The same issue emerged in the 1990s during attempts to date this layer (Haesaerts et al. 1996). The only date close to the expected archaeological age is $21.4 \mathrm{ka} \mathrm{BP}$ (Poz-99994), made on an unimpregnated burnt mammoth bone stored in the archaeological collection. Still, this date when calibrated with $95.4 \%$ probability $(25,980-25,430$ cal BP) does not overlap the period marked by the formerly obtained four radiocarbon dates $(29,360-27,240$ cal BP) (Haesaerts et al. 1996).

\section{Kraków Spadzista Layer 6}

Kraków Spadzista layer 6 yielded the most dates for a Late Gravettian occupation in ECE (Table 10). The dense clustering of mammoth bones in the excavated site is similar to that found in Milovice I, although on a larger scale, again suggesting a rather short interval of human activity consisting of repeated events of mammoth exploitation in a single locus. Indeed, seven of 13 calibrated dates (Figure 5, upper part) are concordant with this suggestion, and situate this phase (Figure 5, lower part) most probably between 27.9 and $27.6 \mathrm{ka}$ cal BP, supporting suggestions from an earlier study of mammoth taphonomy and the mortality profile (Haynes and Klimowicz 2015). Dates of the other samples (outlying the major phase) are difficult to interpret; they may reflect sporadic hunting events, or some may be from contaminated material (although the acceptable values of $\mathrm{C} / \mathrm{N}$ ratio do not indicate significant contamination). 


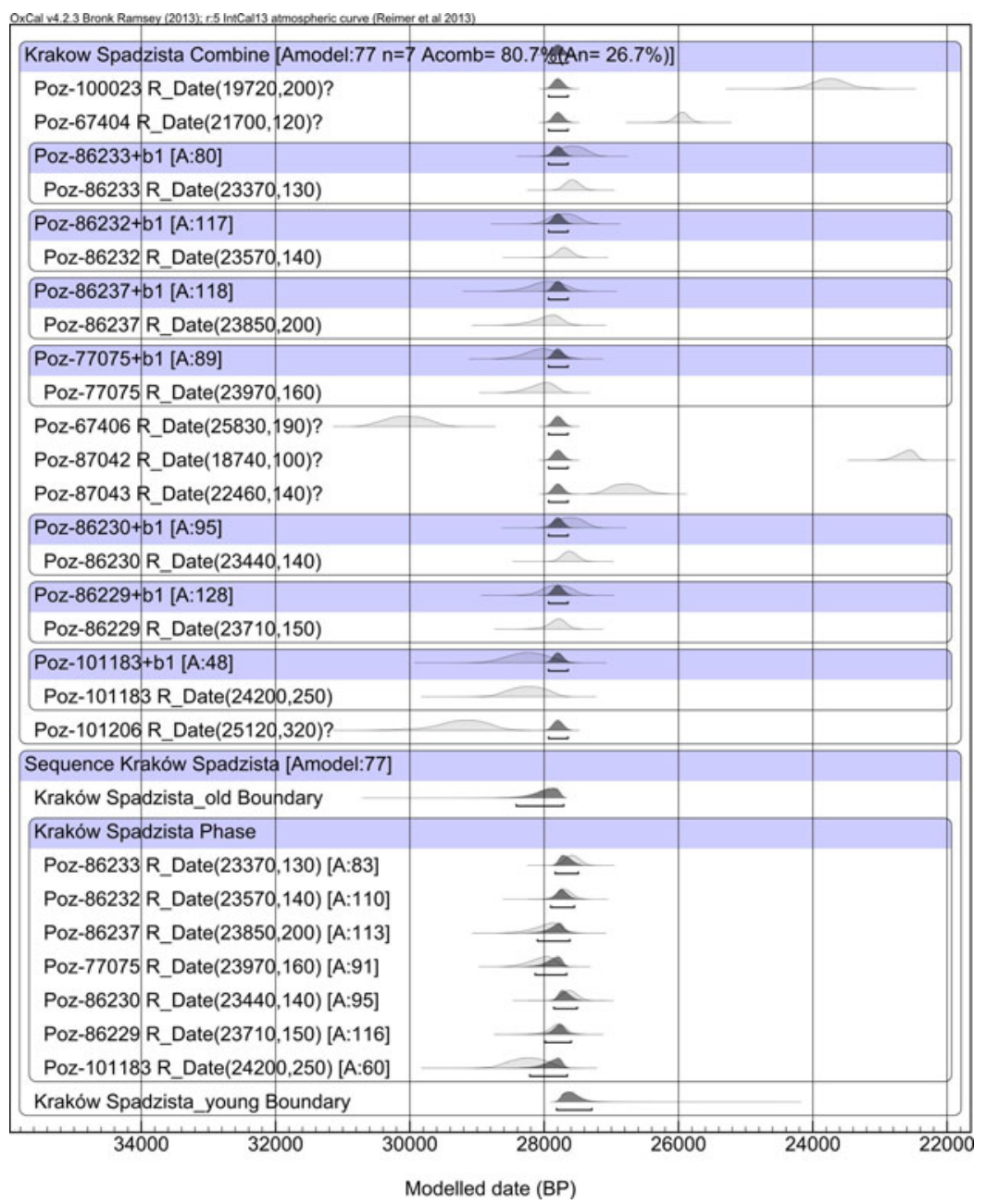

Figure 5 Upper part: combined calibrated ${ }^{14} \mathrm{C}$ dates from Kraków Spadzista, blurred by adding uniform probability distributions 500 years wide $(\mathrm{b} 1=\mathrm{U}(-250,250))$. Six of 13 dates were declared as outliers of the phase. Lower part: Bayesian modeling of phase encompassing dates of seven bones.

\section{Bodrogkeresztúr}

The results from Bodrogkeresztúr are the first acceptable radiocarbon dates for the Upper Palaeolithic human occupation (Table 11). Three of the four samples are dated in a narrow range, between 27.9 and $26.4 \mathrm{ka}$ cal BP. The first date in Table 13 (Poz-95148) is distinctly younger and difficult to interpret, since despite the low collagen extraction yield, the C/N atomic ratio in collagen did not indicate its contamination with ambient carbon. If this date is correct, the time interval encompassing all four dates still corresponds with the Late Gravettian age, which was estimated based on lithic tool typology (Lengyel 2015) (Figure 6). 


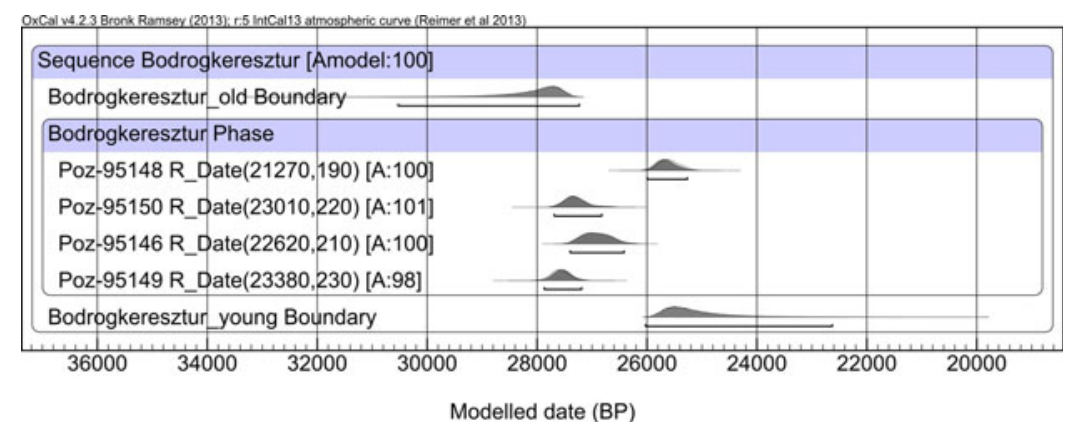

Figure 6 Bayesian modeling of phase encompassing dates of bones from Bodrogkeresztúr.

\section{Pilisszántó I}

The dates for Pilisszántó I Rockshelter lower layer complex are the first radiocarbon results for this site (Table 12). The dates from the lower layer complex, between 30.0 and $28.5 \mathrm{ka}$ cal BP, are older than the age estimated by the biostratigraphy (Dobosi and Vörös 1987; Dobosi and Szántó 2003). These ages are in agreement with the Late Gravettian cultural affiliation (Lengyel 2016).

\section{DISCUSSION AND CONCLUSION}

Radiocarbon chronology for the MUP in ECE seems to show that the region's earliest Late Gravettian dates at Milovice I (30.8 ka cal BP) are contemporaneous with the Pavlovian at least over 1.8 ka years. For instance, Dolní Věstonice I site yielded an abundant inventory of Pavlovian culture (Oliva 2014), including 11 radiocarbon dates obtained with decay counting method except for the only bone date in the series, run on a human femoral diaphysis (Svoboda et al. 2018). The human bone date is the youngest, $22.8 \mathrm{ka} \mathrm{BP}$, while all the others, mainly charcoals associated with the lithic artifacts, are significantly older, 25.7 and $25.9 \mathrm{ka} \mathrm{BP}$. There are three more dates on mammoth molars from the 1924-1925 excavation, 20.4, 24.1 and $25.5 \mathrm{ka} \mathrm{BP}$, which are also somewhat younger than other ${ }^{14} \mathrm{C}$ dates from the site (Nadachowski et al. 2018).

For the moment this chronological overlap is difficult to explain. We suggest three possible explanations: (1) the Pavlovian did overlap the beginning of the Late Gravettian; or (2) the Dolní Věstonice I human occupation recovered in the early- and mid-20th century was a palimpsest deposit that included both Pavlovian and Late Gravettian remains; or (3) the Pavlovian occupations were finely stratified, as shown by the new excavations (Svoboda 2016), and the resolution of the field methods of the early- and mid-20th century was unable to notice tiny archaeological layers. An admixture of Late Gravettian and Pavlovian was noted earlier regarding the Pavlov I site (Verpoorte 2005).

The results reported here suggest that Late Gravettian occupation in ECE occurred between 30 and $26 \mathrm{ka}$ cal BP. We do not find an admixture of bone materials from several occupational periods at the Late Gravettian sites except Kraków Spadzista where solifluction disturbed the archaeological layer. 
Radiocarbon dates of Late Gravettian younger than $22 \mathrm{ka}$ BP most probably are too late to represent the end of that culture. Currently we cannot explain why a few dates are outliers from the archaeological chronology; for instance the radiocarbon ages 19 and $20 \mathrm{ka}$ BP for Lubná VI Late Gravettian assemblage are too young and correspond with the Epigravettian (Lengyel and Wilczyński 2018).

The new dates reported here place the beginning of the Late Gravettian one millennium earlier and its end two millennia earlier than currently assumed in the literature. Unfortunately, the chronological overlap between the end of the Pavlovian and the beginning of the Late Gravettian has not been resolved.

\section{ACKNOWLEDGMENTS}

J. Wilczyński's studies were supported by the National Science Centre, Poland, agreement No. UMO-2015/18/E/HS3/00178. P. Wojtal's studies were supported by the National Science Centre, Poland, agreement No. UMO-2015/17/B/HS3/00165. G. Lengyel's studies were supported by the National Science Centre, Poland, agreement No. UMO-2016/23/P/HS3/ 04034. This project has received funding from the European Union's Horizon 2020 research and innovation programme under the Marie Skłodowska-Curie grant agreement No 665778. We are grateful to Klára Palotás and László Makádi of the Mining and Geological Survey of Hungary for helping to sample Bodrogkeresztúr and Pilisszántó I rockshelter faunal material. We would like to thank G. Haynes, University of Nevada, for correcting the English text.

\section{REFERENCES}

Absolon K. 1945. Die Erforschung der diluvialen Mammutjäger-Station von Unter-Wisternitz in Mähren. Arbeitsbericht über das dritte Grabungsjahr 1926. Selbstverlag.

Antl-Weiser W. 2009. The time of the Willendorf figurines and new results of palaeolithic research in Lower Austria. Anthropologie XLVII(1-2):131-41.

Brock F, Higham T, Ditchfield P, Bronk Ramsey C. 2010. Current pretreatment methods for AMS radiocarbon dating at the Oxford Radiocarbon Accelerator Unit (ORAU). Radiocarbon 52:103-112.

Bronk Ramsey C. 2009. Dealing with outliers and offsets in radiocarbon dating. Radiocarbon 51(3):1023-1045.

Bronk Ramsey C, Higham T, Bowles A, Hedges R. 2004. Improvements to the pretreatment of bone at Oxford. Radiocarbon 46:155-163.

Bronk Ramsey C, Lee S. 2013. Recent and planned developments of the program OxCal. Radiocarbon 55:720-730.

Brugère A, Fontana L.. In: Oliva M 2009. Mammoth origin and exploitation patterns at Milovice (area $\mathrm{G}$ excepted). In: , editor. Milovice: site of the Mammoth People below the Pavlov Hills: The question of mammoth bone structures. Brno. p. 53-105.
Dobosi VT, editor. 2000. Bodrogkeresztúr-Henye (NE Hungary), Upper Palaeolithic site. Hungarian National Museum, Budapest.

Dobosi VT, Szántó Zs. 2003. A gravetti idôszak hagyományos és radiokarbon koradatai. Archaeologiai Értesítő 128:5-16.

Dobosi VT, Vörös I. 1987. The Pilisszántó I. Rock-shelter revision. Folia Archaeologica 38:7-64.

Gábori M. 1954. A pilisszántói kőfülke magdaleni kultúrája és eredete. Archaeológiai Ertesítő 81:3-9.

Goslar T, Czernik J, Goslar E. 2004. Low-energy ${ }^{14} \mathrm{C}$ AMS in Poznań Radiocarbon Laboratory, Poland. Nuclear Instruments and Methods in Physics Research B 223-224:5-11.

Haesaerts P, Damblon F, Bachner M, Trnka G. 1996. Revised stratigraphy and chronology of the Willendorf II sequence, Lower Austria. Archaeologia Austriaca 80:25-42.

Haynes G. 1991. Mammoths, mastodonts and elephants: Biology, behavior and fossil record. Cambridge University Press.

Haynes G, Klimowicz J. 2015. The meaning of the Mammoth age profile from Kraków Spadzista trench B+B1. In: Wojtal P, Wilczyński J, Haynes G, editors. A Gravettian site in southern Poland: Kraków Spadzista, Kraków. p. 159-187. 
Händel M. 2017. The stratigraphy of the Gravettian sites at Krems. Quartär 64:129-155.

Klíma B. 1963. Dolní V\&ebreve;stonice, výsledky výzkumu tábořište lovců mamutů v letech 19471952. Praha.

Kormos T, Lambrecht K. 1915. A pilisszántói kőfülke. Tanulmányok a postglaciális kor geológiája, ősipara és faunája köréből. Földtani Intézet Évkönyve 23:307-498.

Kozłowski JK. 2008. The shouldered point horizon and the impact of the LGM on human settlement distribution in Europe. In: Svoboda J, editor. Petřkovice: on shouldered points and female figurines. The Dolní Věstonice Studies, Vol. 15. Brno: Institute of Archaeology at Brno, Academy of Sciences of the Czech Republic. p. 181-192.

Kozłowski JK. 2013. Raw materials procurement in the Late Gravettian of the Carpathian Basin. In: Mester Zs, editor. The lithic raw material sources and the interregional human contacts in the Northern Carpathian regions. KrakówBudapest: Polska Akademia Umiejętności. p. $63-85$.

Kozłowski JK, van Vliet B, Sachse-Kozłowska E, Kubiak H, Zakrzewska G. 1974. Upper paleolithic site with dwellings of mammoth bones e Cracow, Spadzista street B. Folia Quaternaria 44:1-110.

Kufel-Diakowska B, Wilczyński J, Wojtal P, Sobczyk K. 2016. Mammoth hunting-Impact traces on backed implements from a mammoth bone accumulation at Kraków Spadzista (southern Poland). Journal of Archaeological Science 65:122-133.

Kuzmin YV, Keates SG., 2005. Paleolithic settlement patterns in Siberia derived from radiocarbon records. American Antiquity 70:773-789.

Lengyel G. 2008-2009. Radiocarbon dates of the "Gravettian Entity" in Hungary. Praehistoria 9-10:241-263.

Lengyel G. 2015. Lithic raw material procurement at Bodrogkeresztúre-Henye Gravettian site, northeast Hungary. Quaternary International 359-360: 292-303.

Lengyel G. 2016. Reassessing the Middle and Late Upper Palaeolithic in Hungary. Acta Archaeologica Carpathica 51:47-66.

Lengyel G, Wilczyński J. 2018. The Gravettian and the Epigravettian chronology in eastern central Europe: a comment on Bösken et al. 2017. Palaeogeography, Palaeoclimatology, Palaeoecology 506:265-269.

Longin R. 1971. New method of collagen extraction for radiocarbon dating. Nature 230:241-242.

Moreau L. 2009. Geißenklösterle. Das Gravettien der Schwäbischen Alb im europäischen Kontext. Tübingen: Kerns Verlag.

Moreau L. 2012. Le Gravettien ancien d'Europe centrale revisité: mise au point et perspectives. L'Anthropologie 116:609-638.
Nadachowski A, Lipecki G, Baca M., Żmihorski M, Wilczyński J. 2018. Impact of climate and humans on the range dynamics of the woolly mammoth (Mammuthus primigenius) in Europe during MIS 2. Quaternary Research 90/3:439-456.

Nigst PR, Haesaerts P, Damblon F, Frank-Fellner C, Mallol C, Viola B, Götzinger M, Niven L, Trnka G, Hublin J-J. 2014. Europeans occupied a cold steppe 43,500 years ago. Proceedings of the National Academy of Sciences 111(40):14394-9.

Novák M. 2016. Lithics on the periphery. Variability in assemblages from the southern edge and the Dolní Věstonice IIa sub-site (after 1990). In: Svoboda J, editor, Dolní Veštonice II. Chronostratigraphy, Paleoethnology, Paleoanthropology. The Dolní Vestonice Studies vol 21, Archeologický ústav AV ČR Brno. p. 246-72.

Nuzhnyi DYu, Praslov ND, Sablin MV. 2016. Первый случай подтверждения успешной охоты на мамонта в европе (стоянка Костенки 1, Россия). Электронная библиотека Музея антропологии и этнографии им. Петра Великого (Кунсткамера) PAH [The first case of confirmation of a successful mammoth hunt in Europe (Kostenki 1 parking lot, Russia). Electronic Library of the Museum of Anthropology and Ethnography Peter the Great (Kunstkamera) RAS]. http://www. kunstkamera.ru/lib/rubrikator/05/978-5-88431267-8/. In Russian.

Oliva M. 2007. Gravettien na Moravě - Le Gravettien en Moravie. DABP 1. Brno-Praha.

Oliva M, editor. 2009. Milovice: Site of the Mammoth People below the Pavlov Hills: The question of Mammoth bone structures. Brno: Moravské Zemské Muzeum.

Oliva M. 2014. Dolní Věstonice I (1922-1942) Hans Freising - Karel Absolon - Assien Bohmers. Studies in Anthropology, Palaeoethnology, Palaeontology and Quaternary Geology 37(29). Brno.

Piotrowska N, Goslar T. 2002. Preparation of bone samples in the Gliwice radiocarbon laboratory for AMS radiocarbon dating. Isotopes in Environmental and Health Studies 38(4):267-275.

Reimer PJ, Bard E, Bayliss A, Beck JW, Blackwell PG, Bronk Ramsey C, Buck CE, Cheng H, Edwards RL, Friedrich M, Grootes PM, Guilderson TP, Haflidason H, Hajdas I, Hatté C, Heaton TJ, Hoffman DL, Hogg AG, Hughen KA, Kaiser KF, Kromer B, Manning SW, Niu M, Reimer RW, Richards DA, Scott EM, Southon JR, Staff RA, Turney CSM, van der Plicht J. 2013. IntCal13 and Marine13 radiocarbon age calibration curves $0-50,000$ years cal BP. Radiocarbon 55(4):1869-1887.

Stuiver M, Polach H. 1977. Discussion reporting of ${ }^{14} \mathrm{C}$ data. Radiocarbon 19(3):355-363.

Sinitsyn AA, Stepanova KN, Petrova EA. 2019. New direct evidence of mammoth hunting from Kostenki, primary archaeology. Journal of Interdisciplinary Research 1:149-158. In Russian. 
Soffer O. 1993. Upper Paleolithic adaptation in Central and Eastern Europe and manmammoth interactions. In: Soffer O, Praslov D, editors. From Kostienki to Clovis. New York and London: Plenum Press. p. 31-49.

Steenstrup J. 1889. Mammuthjaeger-Stationen ved Předmost, i det Østerrigke Kronland Mähren, efter et Besøg der i Juni-Juli 1888. Overs. Over d. K.D. Vidensk. Selsk. Forh, 1888. p. 145-212.

Svoboda JA. 2007. The Gravettian on the Middle Danube. Paléo 19:203-220.

Svoboda J. 2016. Settlement units and human fossils. Spatial context, stratigraphies and chronology. In: Svoboda J, editor, Dolní Veštonice II. Chronostratigraphy, paleoethnology, paleoanthropology. The Dolní Vestonice Studies, Vol. 21. Archeologický ústav AV ČR Brno. p. $16-48$.

Svoboda J, Novák M, Sázelová S, Hladilová Š, Škrdla P. 2018. Dolní Věstonice I. Excavations 1990-1993. Přehled výzkumů 59(1):35-67.

Svoboda J, Péan S, Wojtal P. 2005. Mammoth bone deposits and subsistence practices during midupper Palaeolithic in Central Europe: three cases from Moravia and Poland. Quaternary International 126-128:209-221.

Š́da P. 2016. Gravettian lithics assemblages from Lubná (Bohemia). Quaternary International 406(A):120-128.

van Klinken G.J. 1999. Bone collagen quality indicators for palaeodietary and radiocarbon measurements. Journal of Archaeological Science 26:687-695.

Verpoorte A. 2003. Absolute dates for the Bohemian Middle Upper Palaeolithic. Archeologicke rozhledy $\mathrm{LV}(1): 3-9$.

Verpoorte A. 2005. The lithic assemblage of Pavlov I (1954, 1956, 1963, 1964). In: Svoboda J, editor. Pavlov I southeast. A window into the Gravettian lifestyles. The Dolní Vestonice Studies 14, Archeologický ústav AV ČR Brno. p. 75-111.
Vértes L. 1966. The Upper Palaeolithic Site on Mt. Henye at Bodrogkeresztúr. Acta Archaeologica Academiae Scientiarum Hungaricae 18:3-14.

Vörös I. 2000. Macro-mammal remains on Hungarian Upper Pleistocene sites. In: Dobosi VT, editor. Bodrogkeresztúr-Henye (NE Hungary), Upper Palaeolithic site, Hungarian National Museum, Budapest. p. 186-212.

Wankel J. 1890. Ložiska mamutí v Předmosti (in Czech). In: Časopis Vlasteneckého Musejního Spolku 7(1-10):53-64.

Wilczyński J. 2007. The Gravettian and Epigravettian lithic assemblages from Kraków-Spadzista B+B1: dynamic approach to the technology. Folia Quaternaria 77:37-96.

Wilczyński J, Wojtal P, Sobczyk K. 2012. Spatial organization of the Gravettian mammoth hunters site - Kraków Spadzista (southern Poland). Journal of Archaeological Science 39: 3627-4362.

Wilczyński J, Wojtal P, Sobieraj D, Sobczyk K. 2015. Kraków Spadzista trench C2 - new research and interpretations of Gravettian settlement. Quaternary International 359-360:96-113.

Wojtal P, Haynes G, Klimowicz J, Sobczyk K, Tarasiuk J, Wroński S, Wilczyński J. 2019. The earliest direct evidence of mammoth hunting in Central Europe - The Kraków Spadzista site (Poland), Quaternary Science Reviews. https:// doi.org/10.1016/j.quascirev.2019.04.004

Wojtal P, Sobczyk K. 2005. Man and woolly mammoth at the Kraków Spadzista Street (B) taphonomy of the site. Journal of Archaeological Science 32:193-206.

Wojtal P, Sobczyk K, Wilczyński J. 2015. New look at old site. Studies of Kraków Spadzista site 19682013. In: Sázelová S, Novák M, Mizerová A, editors. Forgotten times and spaces. New perspectives in paleoanthropological, paleoetnological and archeological studies. Brno: Masaryk University Press. p. 169-190. 